\title{
Measuring the Density Fluctuation From the Cluster Gas Mass Function
}

\author{
Kazuhiro Shimasaku \\ Department of Astronomy, School of Science, University of Tokyo, Bunkyo-ku, Tokyo 113, Japan \\ and \\ Research Center for the Early Universe, School of Science, University of Tokyo, Bunkyo-ku, Tokyo 113, Japan
}

\begin{abstract}
We investigate the gas mass function of clusters of galaxies to measure the density fluctuation spectrum on cluster scales. The baryon abundance confined in rich clusters is computed from the gas mass function and compared with the mean baryon density in the universe which is predicted by the Big Bang Nucleosynthesis. This baryon fraction and the slope of the gas mass function put constraints on $\sigma_{8}$, the rms linear fluctuation on scales of $8 h^{-1} \mathrm{Mpc}$, and the slope of the fluctuation spectrum, where $h$ is the Hubble constant in units of $100 \mathrm{~km} \mathrm{~s}^{-1} \mathrm{Mpc}^{-1}$. We find $\sigma_{8}=0.80 \pm 0.15$ and $n \sim-1.5$ for $0.5 \leq h \leq 0.8$, where we assume that the density spectrum is approximated by a power law on cluster scales: $\sigma(r) \propto r^{-\frac{3+n}{2}}$. Our value of $\sigma_{8}$ is independent of the density parameter, $\Omega_{0}$, and thus we can estimate $\Omega_{0}$ by combining $\sigma_{8}$ obtained in this study with those from $\Omega_{0}$-dependent analyses to date. We find that $\sigma_{8}\left(\Omega_{0}\right)$ derived from the cluster abundance such as the temperature function gives $\Omega_{0} \sim 0.5$ while $\sigma_{8}\left(\Omega_{0}\right)$ measured from the peculiar velocity field of galaxies gives $\Omega_{0} \sim 0.2-1$, depending on the technique used to analyze peculiar velocity data. Constraints are also derived for open, spatially flat, and tilted Cold Dark Matter models and for Cold + Hot Dark Matter models.
\end{abstract}

Subject headings: cosmology: observations — galaxies: clusters: general 


\section{Introduction}

To measure the spectrum of density fluctuations is one of the key issues in discussing the structure formation in the universe. Clusters of galaxies are suitable objects to measure the spectrum on scales of $\sim 10 h^{-1} \mathrm{Mpc}$, where $h$ is the Hubble constant in units of $100 \mathrm{~km} \mathrm{~s}^{-1} \mathrm{Mpc}^{-1}$. This is because the abundance of clusters is sensitive to the nature of the spectrum, in particular the amplitude, and because the fluctuations on cluster scales can be reliably discussed by linear theory.

Henry \& Arnaud (1991) derived $\sigma_{8}=0.59 \pm 0.02$ and $n=-1.7_{+0.35}^{-0.65}$ from the X-ray temperature function of clusters for $\Omega_{0}=1$ universes, where $\sigma_{8}$ is the rms linear fluctuation on scales of $8 h^{-1} \mathrm{Mpc}, \Omega_{0}$ is the cosmological density parameter, and they assumed that the density spectrum is approximated by a power law in wavenumber as $P(k) \propto k^{n}$ on cluster scales. White, Efstathiou, \& Frenk (1993a) obtained $\sigma_{8} \simeq(0.57 \pm 0.05) \Omega_{0}^{-0.56}$ using the spatial number density of rich clusters. Similar results for $\sigma_{8}$ were also obtained by other authors for open and spatially flat Cold Dark Matter (CDM) models using Henry \& Arnaud's (1991) temperature function data; Eke, Cole, \& Frenk (1996) found $\sigma_{8}=(0.50 \pm 0.04) \Omega_{0}^{-\alpha}$ and Viana \& Liddle (1996) found $\sigma_{8}=0.6 \Omega_{0}^{-\alpha}$; in both estimates $\alpha$ varies from $\sim 0.4$ to $\sim 0.6$ depending on the dark matter model assumed. There is, however, a problem with those measurements of $\sigma_{8}$ that one cannot know $\sigma_{8}$ unless $\Omega_{0}$ is given; in general, the measured quantity is not $\sigma_{8}$ but a combination of $\sigma_{8}$ and $\Omega_{0}$ like $\sigma_{8} \Omega_{0}^{0.6}$. This problem is common to almost all methods for measuring $\sigma_{8}$, such as the one using the peculiar velocity field of galaxies.

In this paper, we measure observationally the fraction of baryons confined in clusters of galaxies to the total baryons in the universe using the cluster gas mass function, and give $\Omega_{0}$-independent measurements of $\sigma_{8}$ by comparing the observed baryon fraction with the theoretical prediction. As will be seen in $\S 3$, the theoretical derivation of the baryon fraction does not depend on $\Omega_{0}$, since the baryon fraction measures essentially the fraction of density fluctuations with an overdensity larger than the critical value. We find $\sigma_{8}=0.80 \pm 0.15$, with the quoted errors including the uncertainties in $h(0.5 \leq h \leq 0.8)$. The power-law index of the spectrum, $n$, is also measured.

The plan of this paper is as follows. In $\S 2$ we derive the cluster gas mass function from the observed sample and compute the fraction of baryons confined in rich clusters. In $\S 3$, we describe an analytic model of gravitational halo formation to predict the fraction of mass confined in dark haloes with a given mass range. In $\S 4$, we estimate $\sigma_{8}$ and $n$. We also derive constraints on three sets of CDM models and a set of Cold + Hot Dark Matter (CHDM) models in $\S 4$. Finally, $\S 5$ summarizes our conclusions.

\section{Data}

In this section, we first derive the gas mass function of clusters from X-ray data, and then estimate the fraction of baryons confined in clusters with a given gas mass range. Ebeling et al. (1996a) constructed an X-ray luminosity function (XLF) of clusters of galaxies from the ROSAT Brightest Cluster Sample, which is an X-ray selected, flux limited sample of 172 clusters compiled from ROSAT All-Sky Survey data. They found that the XLF is well fitted by a Schechter function:

$$
\phi(L) d L=A L^{-\alpha} \exp \left(-\frac{L}{L_{\star}}\right) d L
$$

with $A=(4.58 \pm 0.76) \times 10^{-6} h^{3} \mathrm{Mpc}^{-3} \times(0.25 \times$ $\left.10^{44} h^{-2} \mathrm{erg} \mathrm{s}^{-1}\right)^{\alpha-1}, L_{\star}=(2.23 \pm 0.42) \times 10^{44} h^{-2} \mathrm{erg} \mathrm{s}^{-1}$, and $\alpha=1.78 \pm 0.09$. Here, $L$ is the X-ray luminosity in the $0.1-2.4 \mathrm{keV}$ band.

Ebeling et al. (1996b) presented an X-ray sample of 242 Abell clusters from ROSAT All-Sky Survey data in which X-ray luminosity in the $0.1-2.4 \mathrm{keV}$ band is tabulated. Among those, the gas mass within the Abell (1958) radius ( $\equiv 1.5 h^{-1} \mathrm{Mpc}$ ) is given for 40 clusters in Jones \& Forman (1984) and Arnaud et al. (1992). The Abell radius is close to the typical virialization radius of rich clusters, and thus it is reasonable to suppose that most of the cluster gas is within the Abell radius. From the luminosity and the gas mass data of the forty clusters, we find

$$
\begin{aligned}
M_{\text {gas }}= & (0.34 \pm 0.04) \\
& \times\left(\frac{L}{10^{44}}\right)^{0.65 \pm 0.07}\left(h^{-2.5} 10^{14} M_{\odot}\right),
\end{aligned}
$$

where $L$ is in units of $h^{-2} \mathrm{erg} \mathrm{s}^{-1}$ and $M_{\text {gas }}$ is the gas mass within the Abell radius. When estimating the errors in the coefficients of the $L-M_{\text {gas }}$ relation (eq.[2]), we assume that the observed $M_{\text {gas }}$ for each cluster has an error of $\sigma_{\log M_{\text {gas }}}=0.25$, which is the typical scatter of the data points around equation (2). 
Figure 1 shows the distribution of the forty clusters on the $L-M_{\text {gas }}$ plane.

From the XLF and the $L-M_{\text {gas }}$ relation, we obtain the gas mass function:

$$
\begin{aligned}
& \psi\left(M_{\text {gas }}\right) d M_{\text {gas }} \\
& =(1.28 \pm 0.46) \times 10^{-6} \times\left(\frac{M_{\text {gas }}}{M_{\star}}\right)^{-2.20 \pm 0.15} \\
& \times \exp \left[-\left(\frac{M_{\text {gas }}}{M_{\star}}\right)^{1.54 \pm 0.02}\right] d\left(\frac{M_{\text {gas }}}{M_{\star}}\right)\left(h^{3} \mathrm{Mpc}^{-3}\right),
\end{aligned}
$$

where $M_{\star}=0.57 \times 10^{14} h^{-2.5} M_{\odot}$. This gas mass function is consistent with the (cumulative) gas mass function derived by Burns et al. (1996) using optically selected poor and rich clusters.

We now compute the fraction of baryons confined in rich clusters. This calculation consists of three steps: (1) derive the gas density in the universe contributed from clusters, $\rho_{\mathrm{cl}}^{\text {gas }}$, from equation (3); (2) add to $\rho_{\mathrm{cl}}^{\text {gas }}$ the contribution from the stellar mass of cluster galaxies to obtain the baryon density contributed from clusters, $\rho_{\mathrm{cl}}^{\mathrm{B}}$; $(3)$ compute the baryon fraction $f_{\mathrm{B}} \equiv \rho_{\mathrm{cl}}^{\mathrm{B}} / \rho_{0}^{\mathrm{B}}$, where $\rho_{0}^{\mathrm{B}}$ is the mean baryon density in the universe predicted by the Big Bang Nucleosynthesis.

First, we compute $\rho_{\mathrm{cl}}^{\text {gas }}$ by

$$
\rho_{\mathrm{cl}}^{\mathrm{gas}}=\int_{M_{1}}^{M_{2}} M \psi(M) d M,
$$

where $M_{1}$ and $M_{2}$ are the lower and upper limits of cluster gas mass. Since the XLF and $L-M_{\text {gas }}$ relation are available over $10^{42} \lesssim L \lesssim 10^{45}$ and $10^{43} \lesssim L \lesssim$ $10^{45} h^{-2} \mathrm{erg} \mathrm{s}^{-1}$, respectively, we set $\left(M_{1}, M_{2}\right)=$ $\left(7.6 \times 10^{12}, 1.5 \times 10^{14}\right)\left(h^{-2.5} M_{\odot}\right)$, which corresponds to $\left(L_{1}, L_{2}\right)=\left(10^{43}, 10^{45}\right)\left(h^{-2} \mathrm{erg} \mathrm{s}^{-1}\right)$. Performing the integration in equation (4) 1 , we obtain

$$
\rho_{\mathrm{cl}}^{\text {gas }}=(1.51 \pm 0.32) \times 10^{8} h^{0.5} M_{\odot} \mathrm{Mpc}^{-3},
$$

which is translated into the density parameter $\Omega_{\mathrm{cl}}^{\text {gas }}=$ $(5.45 \pm 1.17) \times 10^{-4} h^{-1.5}$. This value of $\Omega_{\mathrm{cl}}^{\text {gas }}$ agrees roughly with what Persic \& Salucci (1992) obtained for a somewhat narrower mass range of clusters, $\Omega_{\mathrm{cl}}^{\text {gas }} \simeq 3.5 \times 10^{-4} h^{-1.3}$, from an analysis using another XLF derived before ROSAT.

\footnotetext{
${ }^{1}$ Actually, we calculate $\int_{L_{1}}^{L_{2}} M(L) \phi(L) d L$ in order to evaluate the errors in $\rho_{\mathrm{cl}}^{\text {gas }}$ more easily.
}

We fit the observed gas mass function by a power law over the range of $M_{1}$ and $M_{2}$ to find

$$
\psi(M) \propto M^{-3.3 \pm 0.2} .
$$

This power-law index will also be used to put constraints on the density fluctuation spectrum, in particular its slope.

Second, we add the contribution from the stellar mass of galaxies in clusters to $\rho_{\mathrm{cl}}^{\text {gas }}$. According to White et al. (1993b), the mass ratio of the stellar component to the hot gas is $0.2 h^{1.5}$ for the Coma Cluster. Since Coma is a typical rich cluster, we assume that other clusters have the same ratio. Under this assumption, the density of baryons in clusters, $\rho_{\mathrm{cl}}^{\mathrm{B}}$, is calculated by

$$
\rho_{\mathrm{cl}}^{\mathrm{B}}=\rho_{\mathrm{cl}}^{\mathrm{gas}}\left(1+0.2 h^{1.5}\right) .
$$

Finally, let us derive $f_{\mathrm{B}}$. The density parameter of baryons in the universe, $\Omega_{0}^{\mathrm{B}}$, is estimated using the Big Bang Nucleosynthesis. We take Walker et al.'s (1991) estimate: $\Omega_{0}^{\mathrm{B}} h^{2}=0.0125 \pm 0.0025$. We do not adopt recent estimates based on the deuterium abundance in QSO absorption systems, because they are still quite controversial (e.g., Carswell et al. 1996; Rugers \& Hogan 1996; Tytler, Fan, \& Burles 1996; Wampler 1996). Since $\rho_{0}^{\mathrm{B}}=\rho_{0}^{\text {cr }} \Omega_{0}^{\mathrm{B}}\left(\rho_{0}^{\text {cr }} \equiv 3 H_{0}^{2} / 8 \pi G\right.$ is the critical matter density and $H_{0}$ is the Hubble constant), we obtain

$$
f_{\mathrm{B}}=(0.044 \pm 0.013)\left(1+0.2 h^{1.5}\right) h^{0.5} .
$$

The errors are at $1 \sigma$ levels and include both the errors in equation (5) and in $\Omega_{0}^{\mathrm{B}}$.

\section{Model}

In this section we derive analytically the fraction of matter confined in haloes with a given mass range, based on the gravitational halo formation model. The Press-Schechter (1974) theory predicts the number density of haloes with a comoving radius $r$ as

$$
\begin{aligned}
n(r) d r= & -\frac{3}{4 \pi}\left(\frac{2}{\pi}\right)^{\frac{1}{2}} \delta_{\mathrm{c}} \\
& \times \frac{1}{r^{3}} \frac{1}{\sigma^{2}(r)} \frac{d \sigma(r)}{d r} \exp \left[-\frac{\delta_{\mathrm{c}}^{2}}{2 \sigma^{2}(r)}\right] d r,
\end{aligned}
$$

where $\delta_{\mathrm{c}}$ is the critical linear overdensity and $\sigma(r)$ is the rms of the linear density fluctuations in top-hat windows of radius $r ; \sigma_{8}$ is defined as $\sigma\left(8 h^{-1} \mathrm{Mpc}\right)$. 
The fraction of matter in the universe which is confined in haloes with $r_{1} \leq r \leq r_{2}$ is expressed as

$$
f_{\mathrm{M}}=\frac{\int_{r_{1}}^{r_{2}} M(r) n(r) d r}{\rho_{0}} .
$$

Here, $M(r)$ is the total mass of the halo with a comoving radius $r$ and $\rho_{0} \equiv \rho_{0}^{\mathrm{cr}} \Omega_{0}$. We assume that the total mass of haloes is within the Abell radius. Since

$$
M(r)=\frac{4 \pi}{3} \rho_{0} r^{3}
$$

equation (10) is rewritten as

$$
f_{\mathrm{M}}=\int_{r_{1}}^{r_{2}} \frac{4 \pi}{3} r^{3} n(r) d r
$$

We then assume that the baryon-to-total mass ratio of haloes is equal to $\rho_{0}^{\mathrm{B}} / \rho_{0}$. This assumption is reasonable because $N$-body simulations to date have suggested that hydrodynamical effects do not significantly influence the baryon-to-total mass ratio within the Abell radius (e.g., White et al. 1993b; Lubin et al. 1996 and references therein). Thus, a cluster with a baryon mass $M_{\mathrm{B}}$ have a total mass $M_{\mathrm{tot}}=M_{\mathrm{B}} \rho_{0} / \rho_{0}^{\mathrm{B}}$, and thus have a comoving radius:

$$
r=\left(\frac{3 M_{\mathrm{B}}}{4 \pi \rho_{0}^{\mathrm{B}}}\right)^{\frac{1}{3}} .
$$

Equations (9), (12), and (13) show that $f_{\mathrm{M}}$, which is equal to $f_{\mathrm{B}}$ by the assumption above, is computed if $\sigma(r), \rho_{0}^{\mathrm{B}}$, and the lower and upper limits of $M_{\mathrm{B}}$ are given. We take $\delta_{\mathrm{c}}=1.69$ throughout this paper for simplicity, because $\delta_{\mathrm{c}}$ depends on $\Omega_{0}$ and the cosmological constant $\lambda_{0}$ only weakly (e.g., Lilje 1992; Lacey \& Cole 1993).

\section{Results and Discussion}

\subsection{Estimates of the Amplitude and Shape of the Fluctuation}

In this subsection, we assume that the density fluctuation spectrum is a power law on cluster scales:

$$
\sigma(r)=\sigma_{8}\left(\frac{r}{8 h^{-1} \mathrm{Mpc}}\right)^{-\frac{3+n}{2}},
$$

and estimate $\sigma_{8}$ and $n$ from $f_{\mathrm{B}}$ and the slope of the gas mass function.

Figure 2 shows the constraints on $\sigma_{8}$ and $n$. Results are shown for two extreme values of $h$, since the

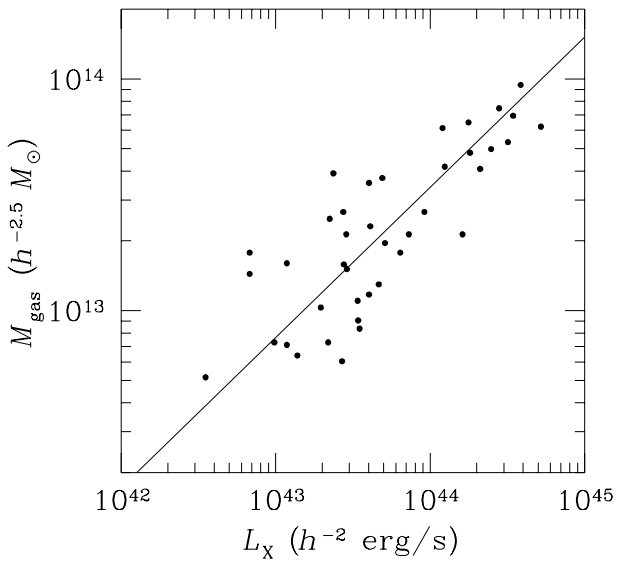

Fig. 1. $-L-M_{\text {gas }}$ relation of forty clusters. Equation (2) is shown as the solid line.

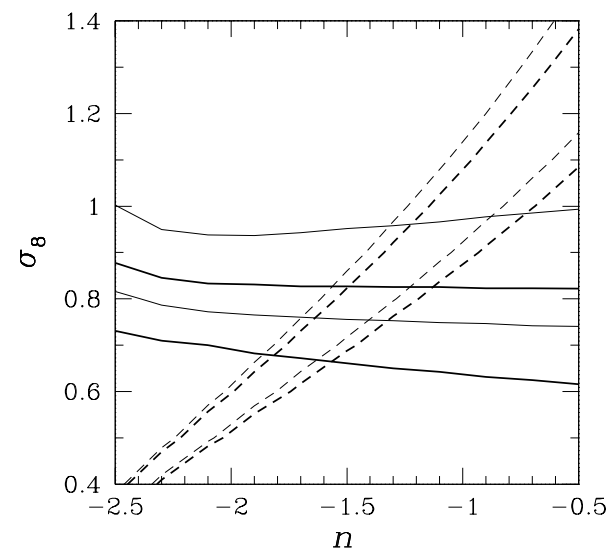

Fig. 2.- Constraints on $\sigma_{8}$ and $n$. The region between the thick (thin) solid lines indicates the range allowed by the baryon fraction $f_{\mathrm{B}}$ for $h=0.5(0.8)$. The region between the thick (thin) dashed lines is for the constraint for $h=0.5$ (0.8) derived from the slope of the gas mass function. 
observed and predicted baryon fractions depend on $h$ (see eqs.[8] and [13]). The two values of $h$ taken here, 0.5 and 0.8 , roughly correspond to the lower and upper limits of recent measurements, respectively (see, e.g., Freedman 1996). The region between the two thick (thin) solid lines indicates the range allowed by the baryon fraction, $f_{\mathrm{B}}$, for $h=0.5(0.8)$; for each $h$, the region corresponds to the $1 \sigma$ range of $f_{\mathrm{B}}$ (eq.[8]). The region between the two thick (thin) dashed lines is for the constraint from the slope of the gas mass function for $h=0.5$ (0.8); for each $h$, the region corresponds to the $1 \sigma$ range of the slope (eq.[6]). In deriving the constraint from the slope of the gas mass function, we fit the theoretical mass function with a power law, $n(M) \propto M^{a}$, over the range of $M$ corresponding to the gas mass range $\left(M_{1}\right.$ and $M_{2}$ in $\left.\S 3\right)$, and compare $a$ with the power-law index of equation (6). We see in Figure 2 two features common to the $h=0.5$ and 0.8 cases. First, $f_{\mathrm{B}}$ places almost the unique constraint on $\sigma_{8}$ irrespective of $n$. This is because $8 h^{-1} \mathrm{Mpc}$ is the typical comoving radius of rich clusters. Second, a higher $\sigma_{8}$ is required for a higher $n$ to reproduce the observed slope of the gas mass function

From Figure 2 we find the allowed values of $\sigma_{8}$ and $n$ to be $\left(\sigma_{8}, n\right)=(0.75 \pm 0.1,-1.5 \pm 0.4)$ for $h=0.5$, and $(0.85 \pm 0.1,-1.3 \pm 0.4)$ for $h=0.8$; a lower $h$ gives a lower $\sigma_{8}$. Adopting $0.5 \leq h \leq 0.8$ as the observational uncertainty in $h$, we can express the range of $\sigma_{8}$ by

$$
\sigma_{8}=0.80 \pm 0.15
$$

In the rest of this subsection, we compare $\sigma_{8}$ obtained above with those from $\Omega_{0}$-dependent analyses to date, and then estimate $\Omega_{0}$. Henry \& Arnaud $(1991)$ derived $\sigma_{8}=0.59 \pm 0.02$ and $n=-1.7_{+0.35}^{-0.65}$ from the cluster temperature function assuming $\Omega_{0}=$ 1. The value of $n$ they obtained agrees very well with what we obtain above. White et al. (1993a) derived $\sigma_{8} \simeq(0.57 \pm 0.05) \Omega_{0}^{-0.56}$ using the cumulative abundance of rich clusters. The basic procedures by Henry \& Arnaud (1991) and White et al. (1993a) to measure $\sigma_{8}$ are the same in the sense that both of them used the cluster abundance and applied the PressSchechter (1974) theory. Thus, White et al.'s (1993a) estimate of $\sigma_{8}$ for arbitrary $\Omega_{0}$ may be regarded as a generalization of Henry \& Arnaud's (1991). Another method for measuring $\sigma_{8}$ is to use the peculiar velocity field of galaxies, though a large inconsistency is seen among recent measurements. Two rep- resentative measurements are those given in Kolatt \& Dekel (1997) and Willick et al. (1996). Kolatt \& Dekel (1997) obtained $\sigma_{8}=(0.71-0.77[ \pm 0.12]) \Omega_{0}^{-0.6}$ from the POTENT analysis (Dekel, Bertschinger, \& Faber 1990; Dekel 1994) of the Mark III peculiar velocity catalog (Willick et al. 1997). Willick et al. (1996) found $\sigma_{8}=(0.34 \pm 0.05) \Omega_{0}^{-0.6}$, applying another technique to the same catalog. One can also measure $\sigma_{8}$ (as a function of $\Omega_{0}$ ) using the redshiftspace anisotropy of galaxy clustering; examples are Fisher et al. (1994) and Cole, Fisher, \& Weinberg (1995), both of which derived similar values to what Willick et al. (1996) obtained 2 .

Since our estimate of $\sigma_{8}$ is independent of $\Omega_{0}$ while those given in the previous papers listed above depend on $\Omega_{0}$ in a way like $\sigma_{8}\left(\Omega_{0}\right)=$ const. $\times \Omega_{0}^{-0.6}$, we can compute $\Omega_{0}$ by comparing them. We take three estimates of $\sigma_{8}\left(\Omega_{0}\right)$ as representatives of the previous ones: those given in White et al. (1993a), Kolatt \& Dekel (1997), and Willick et al. (1996). A comparison between our $\sigma_{8}$ and White et al.'s (1993a) $\sigma_{8}\left(\Omega_{0}\right)$ gives $\Omega_{0}=0.6_{-0.2}^{+0.3}$ (for $h=0.5$ ) and $\Omega_{0}=0.5_{-0.2}^{+0.2}(h=0.8)$. From Kolatt \& Dekel's (1997) measurement we obtain $\Omega_{0}=1.0_{-0.5}^{+0.7}(h=0.5)$ and $\Omega_{0}=0.8_{-0.3}^{+0.5}(h=0.8)$. Finally, Willick et al.'s (1996) $\sigma_{8}\left(\Omega_{0}\right)$ gives $\Omega_{0}=0.3_{-0.1}^{+0.2}(h=0.5)$ and $\Omega_{0}=0.2_{-0.1}^{+0.1}$ $(h=0.8)$. We then compare these values with that computed from the cluster baryon-to-total mass ratio and $\Omega_{0}^{\mathrm{B}}$. White et al. (1993b) found that the baryon-to-total mass ratio for the Coma Cluster is $0.009+0.05 h^{-1.5}$ (the $1 \sigma$ error at a given value of $h$ is $\sim 30 \%$ ). Adopting this ratio as the global value in the universe and using $\Omega_{0}^{\mathrm{B}}=(0.0125 \pm 0.0025) h^{-2}$, we obtain $\Omega_{0}=0.33 \pm 0.1$ for $h=0.5$ and $\Omega_{0}=0.25 \pm 0.1$ for $h=0.8$. These values of $\Omega_{0}$ are in very good agreement with those derived from Willick et al.'s (1996) measurement of $\sigma_{8}\left(\Omega_{0}\right)$, but are smaller than those derived from White et al.'s (1993a) and Kolatt \& Dekel's (1997), though the disagreements are less than $2 \sigma$ levels. To summarize, we find that there exists a region on the $\Omega_{0}-\sigma_{8}$ plane where the three constraints overlap with each other within the observational uncertainties, i.e., the constraints on $\sigma_{8}$ obtained from the gas mass function in this paper,

\footnotetext{
${ }^{2}$ The quantity Fisher et al. (1994) and Cole et al. (1995) measured is not $\sigma_{8}$ but $\beta_{I} \equiv \Omega_{0}^{0.6} / b_{I}$, where $b_{I}$ is the bias parameter of IRAS galaxies. However, as is shown in Willick et al. (1996), one can derive $\sigma_{8}\left(\Omega_{0}\right)$ from $\beta_{I}$ and the observed rms fluctuations of IRAS galaxies on an $8 h^{-1} \mathrm{Mpc}$ scale, assuming that biasing is independent of scale.
} 
on $\sigma_{8}\left(\Omega_{0}\right)$ derived from the $\Omega_{0}$-dependent analyses discussed above, and on $\Omega_{0}$ derived from the cluster baryon-to-total mass ratio and $\Omega_{0}^{\mathrm{B}}$.

\subsection{Constraints on Cosmological Models}

In this subsection, we examine four sets of cosmic structure formation models including (1) open $\left(\lambda_{0}=0\right)$ CDM models with $\Omega_{0}$ the free parameter, (2) spatially flat CDM models with $\Omega_{0}$ the free parameter, (3) tilted CDM models with $n$ the free parameter, and (4) CHDM models with the density parameter of the hot dark matter, $\Omega_{\nu}$, the free parameter. In what follows, $n$ denotes the power-law index of the primordial (i.e., before filtered by the transfer function) density fluctuation spectrum, not the index of the current spectrum on cluster scales. Table 1 summarizes the model parameters. For each set, we constrain the above free parameter by requiring that it matches simultaneously the observed baryon fraction and the 4-year COBE DMR data (Bennett et al. 1996). We take the functional forms of the CDM and CHDM spectra from those given in Kitayama \& Suto (1996) and Ma (1996), respectively (these authors took the form of the CDM spectrum from Bardeen et al. 1986 and corrected it for the baryon component). We do not use the slope of the gas mass function to constrain the models because the CDM and CHDM spectra on cluster scales give power-law indices which are close to those derived in $\S 4.1$. We assume $\Omega_{0}=1$ for the tilted CDM models and the CHDM models for definiteness, although this assumption is apparently inconsistent with the values of $\Omega_{0}$ derived from $\Omega_{0}^{\mathrm{B}}$ and the baryon-to-total mass ratio for Coma (see $\S 4.1$ ).

\section{Open CDM models}

Figure 3 plots the constraints on $\sigma_{8}$ and $\Omega_{0}$ for open CDM models. Results are shown for two extreme values of $h$. The region between the two thick (thin) solid lines is allowed by the baryon fraction, $f_{\mathrm{B}}$, for $h=0.5(0.8)$. The thick (thin) dashed line indicates $\sigma_{8}$ for $h=0.5(0.8)$ which is estimated from the

Table 1: Model parameters.

\begin{tabular}{cccccc}
\hline \hline Model & $h$ & $n$ & $\Omega_{0}$ & $\lambda_{0}$ & $\Omega_{\nu}$ \\
\hline open CDM & $0.5,0.8$ & 1 & varying & 0 & 0 \\
spatially flat CDM & $0.5,0.8$ & 1 & varying & $-\Omega_{0}$ & 0 \\
tilted CDM & 0.5 & varying & 1 & 0 & 0 \\
CHDM & 0.5 & 1 & 1 & 0 & varying \\
\hline
\end{tabular}

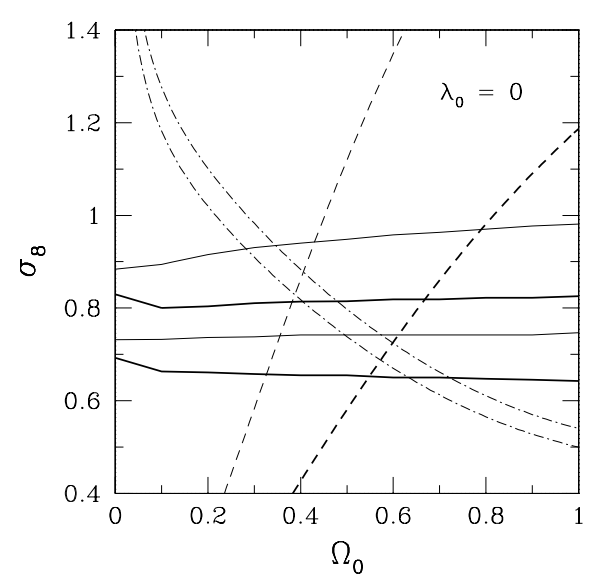

Fig. 3.- Constraints on $\sigma_{8}$ and $\Omega_{0}$ for open CDM models. The region between the thick (thin) solid lines indicates the range allowed by the baryon fraction $f_{\mathrm{B}}$ for $h=0.5(0.8)$. The value of $\sigma_{8}$ given by the 4-year COBE data for $h=0.5(0.8)$ is shown by the thick (thin) dashed line. The region between the dash-dotted lines indicates the constraint derived by Kitayama \& Suto (1997).

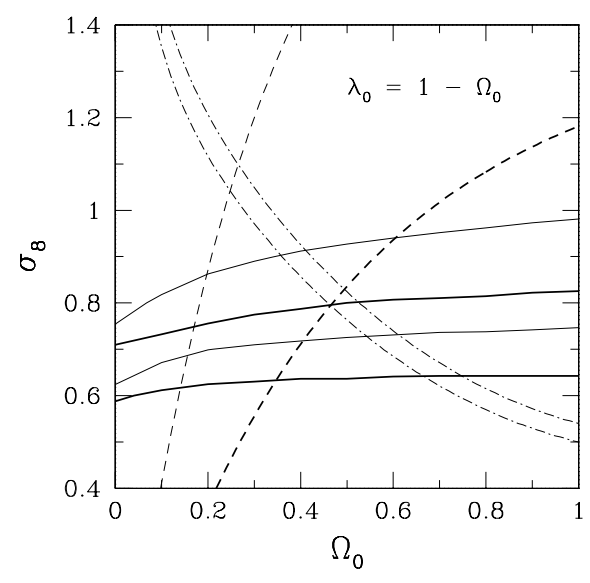

Fig. 4.- Same as Figure 4, but for spatially flat CDM models. 


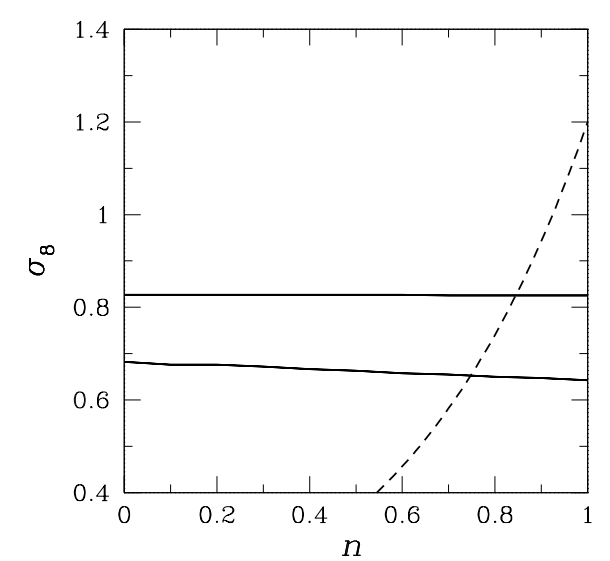

Fig. 5.- Constraints on $\sigma_{8}$ and $n$ for tilted CDM models. The region between the thick solid lines indicates the range allowed by the baryon fraction $f_{\mathrm{B}}$. The value of $\sigma_{8}$ given by the 4-year $C O B E$ data is shown by the thick dashed line.

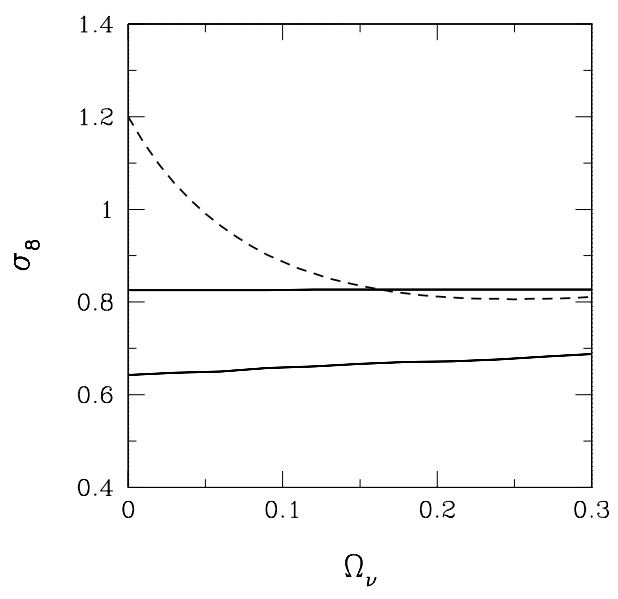

Fig. 6.- Constraints on $\sigma_{8}$ and $\Omega_{\nu}$ for CHDM models. The meaning of the curves is the same as in Figure 5 . 4-year COBE data (Bennett et al. 1996); we use the formulae given in Bunn \& White (1996) to derive $\sigma_{8}$ for open and spatially flat CDM models. For $h=0.5$, $\Omega_{0} \simeq 0.55-0.65$ is required to reproduce simultaneously the baryon fraction and the COBE data. For $h=0.8$, the allowed range is $\Omega_{0} \simeq 0.35-0.45$. In any case, $\Omega_{0}=1$ models are disfavored irrespective of $h$. The allowed values of $\Omega_{0}$ obtained here agree with those recently obtained for scale-invariant CDM models using the cluster abundance; Bahcall \& Cen (1993) found that low-density $\left(\Omega_{0} \sim 0.25-0.35\right)$, low-bias $\left(\sigma_{8} \sim 1-0.8\right)$ CDM models, with or without $\lambda_{0}$, are consistent with the observed cluster mass function; Kitayama \& Suto (1996) found that if $\Omega_{0} \sim 0.2-0.5$ and $h=0.7$ are taken, CDM models with the fluctuation spectrum normalized by the $C O B E$ data reproduce the observed cluster temperature function irrespective of $\lambda_{0}$; Liddle et al.(1996) concluded that for $h>0.6$, open CDM models for which the fluctuation spectrum is normalized by the $C O B E$ data match the observations of galaxies, clusters, and damped Ly $\alpha$ systems considered in their paper only for the range $0.35<\Omega_{0}<0.55$; Kitayama \& Suto (1997) derived constraints on $\sigma_{8}$ and $\Omega_{0}$ for CDM models from the observed $\log N-\log S$ relation of X-ray clusters. As an example we plot the result obtained by Kitayama \& Suto (1997) in Figure 3; the region between the dash-dotted lines indicates the allowed range obtained by Kitayama \& Suto (1997) 3 . We find that for each value of $h$, there is an area on the $\Omega_{0}-\sigma_{8}$ plane where the model reproduces both the baryon fraction, the $\log N-\log S$ relation, and the COBE data. The constraints on $\Omega_{0}$ obtained here are also roughly consistent with those derived from the cluster baryon-tototal mass ratio and $\Omega_{0}^{\mathrm{B}}$ in $\S 4.1$.

\section{Spatially flat CDM models}

Figure 4 shows the constraints on $\sigma_{8}$ and $\Omega_{0}$ for spatially flat CDM models. The meaning of the lines is the same as in Figure 3. For $h=0.5, \Omega_{0} \simeq$ $0.35-0.45$ is required, while as low as $\Omega_{0} \simeq 0.15-0.2$ is required for $h=0.8$. These values of $\Omega_{0}$ are also consistent with the previous estimates mentioned above. As an example we compare our results with that derived by Kitayama \& Suto (1997) in Figure 4.

\footnotetext{
${ }^{3}$ Kitayama \& Suto's (1997) result plotted here is for $h=0.7$, since they gave an analytic formula for the constraint on $\sigma_{8}$ and $\Omega_{0}$ only for $h=0.7$ models. However, figure 3 in their paper shows that the result is robust against varying $h$ from 0.5 to 0.8 .
} 
For $h=0.5$, models with $\left(\Omega_{0}, \sigma_{8}\right) \simeq(0.45,0.8)$ match both the baryon fraction, the $\log N-\log S$ relation, and the $C O B E$ data, though no such set of $\left(\Omega_{0}, \sigma_{8}\right)$ is found for $h=0.8$.

\section{Tilted CDM models}

Figure 5 shows the results for tilted CDM models. We fix $h=0.5$ so that the age of the universe is $13 \mathrm{Gyr}$ and is consistent with that predicted from globular clusters with $(1-2) \sigma$ levels. The region between the two solid lines indicates the constraint from the baryon fraction. The dashed line shows $\sigma_{8}$ which is estimated from the 4-year $C O B E$ data (Ma 1996). We find that $n \simeq 0.75-0.85$ is required to match both the baryon fraction and the $C O B E$ data. Liddle et al. (1995) found that $h=0.5$ tilted models for which the spectrum is normalized by the $C O B E$ data seem to be marginally consistent with the observations considered in their paper if $n \sim 0.6-0.7$ is taken, which is slightly smaller than the value we obtain here.

\section{CHDM models}

Finally, CHDM models are examined in Figure 6. The meaning of the lines is the same as in Figure 5. We find that $\Omega_{\nu} \gtrsim 0.15$ is required to reproduce the baryon fraction and the $C O B E$ data. This result is consistent with what Liddle et al. (1995) obtained; they found that when $h=0.5$ and $n=1$ are taken, $\Omega_{\nu} \sim 0.2-0.3$ models with the fluctuation spectrum normalized by the $C O B E$ data match the observation.

\section{Conclusions}

We have constructed observationally the gas mass function of clusters of galaxies to measure the fraction of baryons confined in clusters to the total baryons in the universe. Comparing this baryon fraction and the slope of the gas mass function with the prediction by the gravitational halo formation model, we have found $\sigma_{8}=0.80 \pm 0.15$ and $n \sim-1.5$ for $0.5 \leq h \leq 0.8$. Our value of $\sigma_{8}$ is independent of $\Omega_{0}$, and thus we can estimate $\Omega_{0}$ from the present result and previous ones in which $\sigma_{8}$ was obtained as a function of $\Omega_{0}$. We have found that $\sigma_{8}\left(\Omega_{0}\right)$ derived from the cluster abundance gives $\Omega_{0} \sim 0.5$ while $\sigma_{8}\left(\Omega_{0}\right)$ measured from the peculiar velocity field of galaxies gives $\Omega_{0} \sim 0.2-1$, depending on the technique used to analyze the peculiar velocity data. We have also examined four sets of cosmic structure formation mod- els and have found that the following models match both the observed baryon fraction and the $C O B E$ data; open CDM models with $\Omega_{0} \simeq 0.55-0.65$ (for $h=0.5)$ and $\Omega_{0} \simeq 0.35-0.45(h=0.8)$; spatially flat CDM models with $\Omega_{0} \simeq 0.35-0.45(h=0.5)$ and $\Omega_{0} \simeq 0.15-0.2(h=0.8) ;$ tilted CDM models with $n \simeq 0.75-0.85$; CHDM models with $\Omega_{\nu} \gtrsim 0.15$.

We thank Yasushi Suto and Tetsu Kitayama for a useful discussion on the Press-Schechter theory and for valuable suggestions. This research was supported in part by the Grants-in-Aid by the Ministry of Education, Science, Sports and Culture of Japan (07CE2002) to RESCEU (Research Center for the Early Universe). 


\section{REFERENCES}

Abell, G. O. 1958, ApJS, 3, 211

Arnaud, M., Rothenflug, R., Boulade, O., Vigroux, L., \& Vangioni-Flam, E. 1992, A\&A, 254, 49

Bahcall, N. A. \& Cen, R. 1993, ApJ, 407, L49

Bardeen, J. M., Bond, J. R., Kaiser, N., \& Szalay, A. S. 1986, ApJ, 304, 15

Bennett, C. L., et al. 1996, ApJ, 464, L1

Bunn, E. F. \& White M. 1996, astro-ph/9607060

Burns, J. O. et al. 1996, ApJ, 467, L49

Carswell, R. F. et al. 1996, MNRAS, 278, 506

Cole, S., Fisher, K. B., \& Weinberg, D. H. 1995, MNRAS, 275, 515

Dekel, A., Bertschinger, E., \& Faber, S. M. 1990, ApJ, 364,349

Dekel, A. 1994, ARA\&A, 32, 371

Ebeling, H., Allen, S. W., Crawford, C. S., Edge, A. C., Fabian, A. C., Böhringer, H., Voges, W., \& Huchra, J. P., 1996a, in Röntgenstrahlung from the Universe, in press

Ebeling, H., Voges, W., Böhringer, H., Edge, A. C., Huchra, J. P., \& Briel, U. G. 1996b, MNRAS, 281, 799

Eke, V. R., Cole, S., \& Frenk, C. S. 1996,MNRAS, submitted

Fisher, K. B., Davis, M., Strauss, M. A., Yahil, A., \& Huchra, J. P. 1994, MNRAS, 267, 927

Freedman, W. L. 1996, in Critical Dialogues in Cosmology, ed. N. Turok (Singapore: World Scientific), in press (astro-ph/9612024)

Henry, J. P. \& Arnaud, K. A. 1991, ApJ, 372, 410

Jones, C. \& Forman, W. 1984, ApJ, 276, 38

Kitayama, T. \& Suto, Y. 1996, ApJ, 469, 480

Kitayama, T. \& Suto, Y. 1997, ApJ, submitted

Kolatt, T. \& Dekel, A. 1997, ApJ, in press

Lacey, C. \& Cole, S. 1993, MNRAS, 262, 627
Liddle, A. R., Lyth, D. H., Schaefer, R. K., Shafi, Q., \& Viana, P. T. P. 1995, astro-ph/9511057

Liddle, A. R., Lyth, D. H., Roberts, D., \& Viana, P. T. P. 1996, MNRAS, 278, 644

Lilje, P. B. 1992, ApJ, 386, L33

Lubin, M. L., Cen, R., Bahcall, N. A., \& Ostriker, J. P. 1996, ApJ, 460, 10

Ma, C.-P. 1996, ApJ, 471, 13

Persic, M. \& Salucci, P. 1992, MNRAS, 258, 14p

Press, W. H. \& Schechter, P. 1974, ApJ, 187, 425

Rugers, M. \& Hogan, C. J. 1996, ApJ, 459, L1

Tytler, D., Fan, X. M., \& Burles, S. 1996, Nature, 381,207

Viana, P. T. P. \& Liddle, A. R. 1996 MNRAS, 281, 323

Walker, T. P., Steigman, G., Schramm, D. N., Olive, K. A., and Kang, H.-S. 1991, ApJ, 376, 51

Wampler, E. J. 1996, Nature, in press

White, S. D. M., Efstathiou, G., \& Frenk, C. S. 1993a, MNRAS, 262, 1023

White, S. D. M., Navarro, J. F., Evrard, A. E., \& Frenk, C. S. 1993b, Nature, 366, 429

Willick, J. A., Strauss, M, A., Dekel, A., \& Kolatt, T. 1996, ApJ, submitted (astro-ph/9612240)

Willick, J. A., Courteau, S., Faber, S. M., Burstein, D., Dekel, A., \& Strauss, M. A. 1997, ApJS, in press (astro-ph/9610202)

This 2-column preprint was prepared with the AAS LATEX macros $\mathrm{v} 4.0$. 